\title{
Distância percorrida em indivíduos hipertensos: estudo de corte transversal
}

\author{
Distance travelled in hypertensive subjects: \\ cross-sectional study
}

\author{
Jaqueline dos Santos Oliveira', Ana Lúcia Barbosa Góes²
}

1Autora para correspondência. Universidade Federal da Bahia. Salvador, Bahia, Brasil. ORCID: 0000-0002-0383-2019. fisio.jaqueline@outlook.com ¿Universidade Federal da Bahia, Escola Bahiana de Medicina e Saúde Pública. Salvador, Bahia, Brasil. ORCID: 0000-0003-2486-0876.goes.ana@ufba.br

RESUMO I INTRODUÇÃO: A hipertensão arterial sistêmica é uma condição clínica ocasionada por diversos fatores que acarretam na elevação dos níveis de pressão arterial de forma sustentada, sendo importante avaliar a capacidade funcional desses indivíduos, visando ter-se um diagnóstico precoce, além de ser um meio de prevenção das repercussões da hipertensão. Um dos instrumentos utilizados é o teste de caminhada de seis minutos (TC6) que é executado em nível submáximo, possibilitando a análise das respostas dos sistemas envolvidos durante as atividades. OBJETIVOS: Verificar a distância percorrida dos indivíduos hipertensos. MATERIAIS E MÉTODOS: Estudo de corte transversal, com população de hipertensos, entre 30 e 60 anos. O TC6 foi realizado de acordo com protocolo padronizado pela ATS e calculada a distância prevista através da equação preditiva de Enright e Sherrill. Foi utilizado o teste T de Student para comparação das médias de distância e calculado o percentual da diferença entre os valores percorridos e previstos. RESULTADOS: 44 sujeitos de ambos

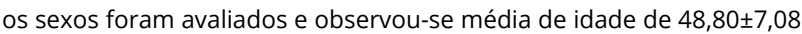
anos, 56,8\% dos indivíduos apresentaram pressão controlada, 81,8\% estavam em uso regular da medicação anti-hipertensiva. A média da distância obtida nos indivíduos que alcançaram os valores previstos foi de $503 \pm 38,6$ metros. Sendo que a maioria dos indivíduos percorreram em média $86,3 \pm 7,2 \%$ da distância prevista. CONCLUSÃo: Indivíduos com hipertensão arterial apresentam diminuição da distância percorrida, independente da pressão arterial estar controlada ou não.

PALAVRAS-CHAVE: Hipertensão. Teste de caminhada de seis minutos. Capacidade funcional.
ABSTRACT | INTRODUCTION: Systemic arterial hypertension is a clinical condition caused by several factors that cause the elevation of blood pressure levels in a sustainable way, it is important to assess the functional capacity of these individuals, aiming to have an early diagnosis, in addition being a means of preventing the effects of hypertension. One of the instruments used is the six-minute walk test (6MWT) that runs in the submaximal level, enabling the analysis of the responses of the systems involved during the activities. OBJECTIVES: To determine the distance travelled of hypertensive individuals. MATERIALS AND METHODS: A cross-sectional study, with a population of hypertensive patients, between 30 and 60 years. The TC6 was performed according to a standardized protocol by the ATS and calculated the distance provided by the predictive equation of Enright and Sherril. The Student T test was used for comparison of the average distance and calculated the percentage difference between the values driven and laid down. RESULTS: 44 subjects of both genders were assessed, with an average age of $48.80 \pm 7.08$ years, $56.8 \%$ of the individuals presented controlled pressure, $81.8 \%$ were in regular use of anti-hypertensive medication. The average distance obtained in individuals who have achieved the expected values was $503 \pm 38.6$ meters. The majority of individuals have traveled on average $86.3 \pm 7.2 \%$ of the expected distance. CONCLUSION: Individuals with arterial hypertension have distance travelled reduced, whether blood pressure is controlled or not.

KEYWORDS: Hypertension. Six-minute walk test. Functional capacity. 


\section{Introdução}

A hipertensão arterial sistêmica (HAS) é uma condição clínica ocasionada por diversos fatores que acarretam na elevação dos níveis pressóricos de forma sustentada da pressão arterial (PA) ${ }^{1}$. Devido à alta prevalência e baixas taxas de controle, é considerado um fator de risco modificável para doenças cardiovasculares (DCV), com custos diretos e indiretos estimados em US\$ 46,4 bilhões em 20112. Dentre os fatores de risco estão idade, sexo e etnia, excesso de peso e obesidade, ingestão de sal, ingestão de álcool, sedentarismo, fatores econômicos e genéticos ${ }^{1}$.

No Brasil, HAS acomete cerca de 32,5\% (36 milhões) de indivíduos adultos, tendo forte contribuição para $50 \%$ dos óbitos por DCV, ocasionando elevado impacto na perda de rendimento do trabalho e da renda familiar, estimada em US\$ 4,18 bilhões entre 2006 e $2015^{1}$. Além disso, as DCV têm constituído o principal gerador de óbitos. Em 2017, houve 92.837 óbitos por doenças do aparelho circulatório. Por volta de 2010 a 2016, pôde-se observar que ocorreu um declínio nas taxas de mortalidade cardiovascular, de 1.400 para 1.021 relacionados à hipertensão, respectivamente ${ }^{3}$.

A representação das habilidades mentais, capacidade de efetuar atividades de vida diárias (AVD's), e realizar tarefas sem auxilio refere-se à capacidade funcional (CF). Quando um indivíduo tem redução do nível de atividade física, e consequentemente da CF, compromete a realização das AVD's. A incapacidade funcional remete a dependência de um indivíduo em executar as atividades cotidianas, podendo relacionar-se à redução no desempenho físico (DF) ${ }^{4,5}$. Modificações na CF são preditoras de morbidade e mortalidade em portadores de doenças crônicas, ao passo que descoberta antecipadamente pode controlar a evolução da HAS e suas repercussões ${ }^{6,7}$. Essa capacidade pode ser avaliada mediante utilização de diversos instrumentos, tais como: teste ergométrico (TE), testes de exercício cardiopulmonar, teste do banco, testes de distância bidirecional, conhecido como ShuttleWalk Test e o teste de caminhada de seis minutos (TC6) ${ }^{8}$.

O TE é entendido como sendo um recurso aceito de forma universal para diagnosticar DCV, sendo usado para determinar o prognóstico na avaliação da resposta terapêutica, da tolerância ao esforço e de sintomas compatíveis com arritmias ao exercício, possibilitando uma avaliação segura e precisa para a prática de atividade física regular. Por se tratar de um teste que possui um custo elevado, não possuindo disponibilidade no Sistema Único de Saúde (SUS), o teste de caminhada de seis minutos (TC6) é de baixo custo e o mais utilizado?.

Esse teste é usado na prática clínica desde a década de 60 , sendo considerado como um teste submáximo que examina globalmente vários sistemas envolvidos durante a prática de exercícios, tais como musculoesquelético, cardiovascular e respiratório. Tem por objetivo estipular a distância percorrida em um percurso plano, além de, retratar corretamente a capacidade funcional dos indivíduos em executar suas tarefas do dia-a-dia ${ }^{10,11}$.

Considerando a existência de alterações da CF em cardiopatas, é possível que este parâmetro possa estar alterado na doença hipertensiva. O controle da HAS bem como seu tratamento são essenciais para reduzir eventos cardiovasculares, e uma vez que se observe que indivíduos com hipertensão apresentam algum impacto na capacidade funcional ${ }^{6}$, identificar precocemente impactos na CF pode funcionar como fator prognóstico de morbimortalidade. Investigar essa associação pode ser um caminho de prevenção das repercussões da HAS, diagnóstico precoce, e consequentemente orientar mudanças nos hábitos de vida. Portanto o presente estudo tem como objetivo verificar a distância percorrida dos indivíduos hipertensos.

\section{Metodologia}

Trata-se de um estudo de corte transversal, através de uma de análise em banco de dados cuja coleta foi realizada no Ambulatório Docente Assistencial da Bahiana (ADAB) e no Complexo Comunitário Vida Plena (CCVP), vinculados à Escola Bahiana de Medicina e Saúde Pública, realizado no período de março de 2013 até dezembro 2017.

\section{Critérios de Inclusão}

Indivíduos com diagnóstico médico de Hipertensão Arterial Sistêmica (HAS) (PAS $\geq 140 \mathrm{mmHg}$ e/ou $P A D \geq 90 \mathrm{mmHg}$ ) validado por repedidas aferições em condições ideais, em duas ou mais ocasiões, e confirmado por medições fora do consultório (MAPA) ${ }^{12}$, 
índice de massa corporal (IMC) até 34,9 Kg/m2, em uso regular de medicação anti-hipertensiva quando indicada, com faixa etária entre 30-60 anos e de ambos os sexos e residentes em região metropolitana.

\section{Critérios de Exclusão}

Alterações musculoesqueléticas ou neuromusculares que limitem significativamente a caminhada, indivíduos fumantes, doenças respiratórias (asma, bronquite, pneumonia recente - últimos 6 meses), alergias, alterações mentais, depressão, insuficiência renal, gravidez e Diabetes Mellitus associadas à HAS e história de eventos cardiovasculares prévios (infarto do miocárdio, insuficiência cardíaca, angina instável, doença arterial periférica) e problemas ortopédicos prévios, detectados em prontuário ou com diagnóstico referido.

\section{Instrumentos Utilizados}

$\mathrm{Na}$ avaliação foram aplicados os seguintes instrumentos: questionário com informações pessoais e dados sócio-demográficos, hábitos de vida e saúde, dados antropométricos, peso e estatura.

Para realização do TC6 foi utilizado: corredor de $30 \mathrm{~m}$, cronômetro, medidor de pressão arterial digital (Marca Omron, modelo HEM-742), oxímetro de pulso (Marca MD 300C1), trena, dois cones de trânsito, fita adesiva, uma cadeira e a escala de Borg modificada, para mensurar a percepção subjetiva do esforço.

Para realização do TC6, de acordo com protocolo padronizado,os indivíduos receberam instruções para deambular sozinho em um tempo de seis minutos, fazendo uso de um corredor de 30 metros de comprimento, com marcações a cada 3 metros sem obstáculos no percurso, realizando uma curva onde estivesse demarcado com um cone, seguindo seu próprio ritmo. O examinador utilizou palavras de incentivo, tais como "Você está indo bem" ou "Continue, bom trabalho"de forma neutra e padronizada no segundo e quarto minutos do teste ${ }^{13,14}$.

Orientações foram passadas aos indivíduos, como não conversar durante a realização do teste, deambular devagar ou até mesmo interromper o teste caso sentisse algum desconforto, tais como dispnéia, dor torácica, tontura ou dor nas pernas. A PA foi aferida no começo e ao final do teste, a frequência cardíaca (FC), a saturação de oxigênio (SpO2), assim como a escala modificada de dispnéia de BORG foram conferidas ao iniciar, durante e ao final dos seis minutos. O percurso foi mostrado ao indivíduo pelo examinador ${ }^{13,15}$.

$\mathrm{Na}$ literatura foram encontradas algumas equações de referência para a previsão da distância percorrida no TC6, e foi aplicada para definir o percentual da diferença entre os valores obtidos e previstos ${ }^{6,16}$. Foi utilizada a equação de Enright e Sherrill ${ }^{15}$.

\section{Cálculo Amostral}

Tomando-se base o estudo de Dias et al. ${ }^{11}$, em que foi encontrado um desvio padrão de distância percorrida no grupo controle em torno de 45 metros, o tamanho amostral foi calculado para comparação pareada de duas médias, considerando um desvio padrão da distância percorrida de 10 metros para a distância prevista e de 45 metros para a distância obtida, com diferença entre as médias dos grupos de $20 \%$, nível de significância de $5 \%$, poder do teste de $80 \%$, em hipótese bicaudal, totalizando 34 pares de indivíduos com 68 observações. O cálculo foi realizado na calculadora WINPEPI® (Published in Epidemiologic Perspectives \&Innovations).

\section{Análise dos Dados}

As variáveis analisadas nesse estudo foram: idade (anos), sexo (dicotômica), estado civil (casado/união estável, separado/divorciado, solteiro, viúvo), escolaridade (anos de estudo, subdividida em 1-4 anos, 5-8 anos, 9-11 anos e 12 ou mais anos), cor da pele (segundo IBGE: preta, branca, parda, amarela, indígena, outra), tabagismo (nunca fumou, fumante, ex-fumante), consumo de bebidas alcoólicas (dicotômica). Para a realização do TC6 as variáveis analisadas foram: distância percorrida (metros), PA (mmHg), FC (bpm), FR (ipm), SpO2 (porcentagem) e percepção do esforço (numérica). 
Foi empregada média e desvio-padrão para realização da análise descritiva das variáveis quantitativas idade (anos), capacidade funcional (distância percorrida), PA, FC, FR e percepção do esforço uma vez que a distribuição foi normal. Variáveis categóricas sexo, estado civil, escolaridade, cor da pele, tabagismo, consumo de bebida alcoólica foram descritas em valores absolutos e em porcentagem.

Para verificar a normalidade da amostra foi realizado o teste de Shapiro-Wilk na variável de desfecho. A variável capacidade funcional foi recategorizada a partir dos valores preditos: alcançou a distância prevista quando a distância obtida foi igual ou maior que a distância prevista; ou não alcançou a distância prevista quando os valores obtidos foram abaixo dos valores preditos, e foi aplicado o teste T de Student para comparação das médias de distância percorrida, além disso, foi analisado o percentual da diferença entre os valores percorrido e previsto. Os dados referentes à pesquisa quantitativa foram organizados utilizando o programa Statistical Package for Social Sciences (SPSS), versão 17.0 para Windows. O banco de dados e análise ficará em posse dos pesquisadores até o término da pesquisa.

\section{Aspectos Éticos}

O presente artigo foi aprovado pelo Comitê de Ética em Pesquisa da Escola Bahiana de Medicina e Saúde Pública, CAAE $n^{\circ}$ 16952113.5.0000.5544. Para participar da pesquisa era preciso ler e assinar o Termo de Consentimento Livre e Esclarecido (TCLE), adaptado conforme o perfil dos participantes. Esse estudo também está registrado no Clinical Trials sob protocolo NCT 02401516.

\section{Resultados}

A seleção dos participantes quanto aos critérios de elegibilidade foi composta por 200 indivíduos, destes foram excluídos 150 por não preencherem os critérios de inclusão. Dos 50 indivíduos aptos, 6 recusaram a participar do estudo. A amostra foi composta por 44 participantes (Figura 1).

Figura 1. Fluxograma para definição dos participantes do estudo

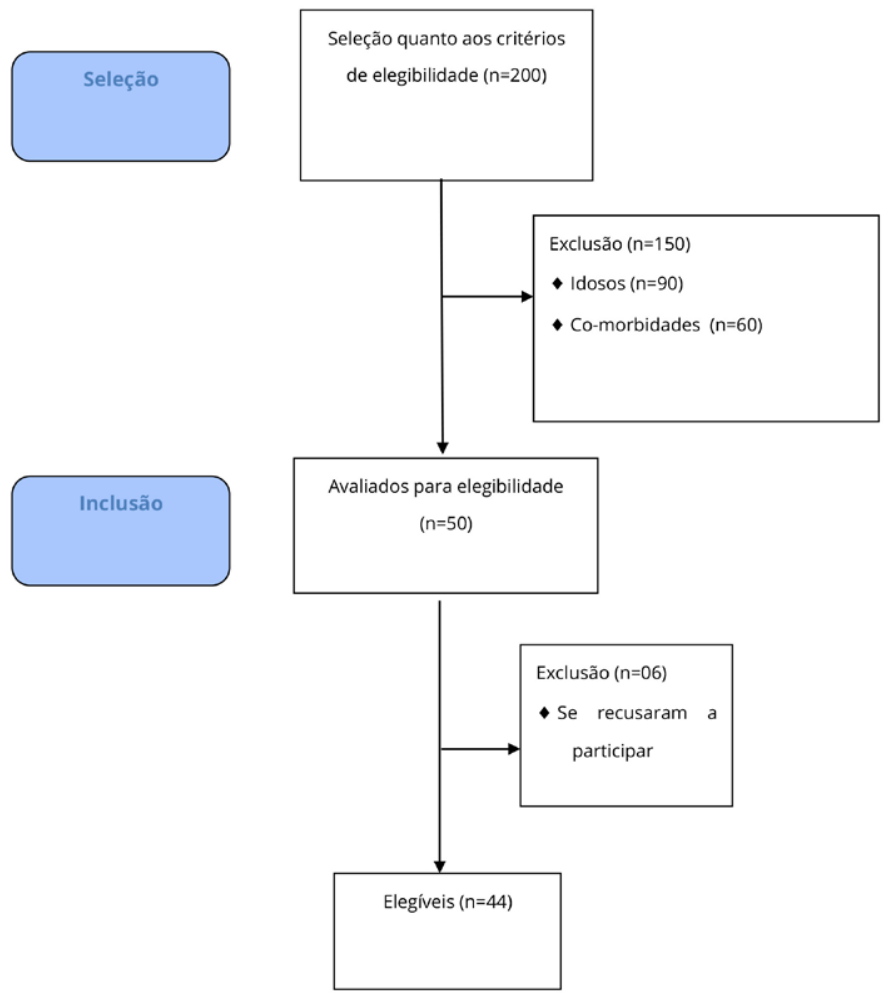


A média de idade da amostra foi de $48,80 \pm 7,08$ anos, média de altura $161,16 \pm 8,18 \mathrm{~cm}$, média de peso $75,70 \pm 10,63 \mathrm{Kg}$, predominância do sexo feminino (75\%), casados (56,8\%), com bom nível de escolaridade com 12 anos ou mais de estudo (63,6\%), cor da pele preta (50\%) (Tabela 1).

Tabela 1. Características da amostra quanto aos dados sociodemográficos, antropométricos e clínicos. Salvador-Ba. 2018 ( $n=44$ )

\begin{tabular}{|c|c|}
\hline Variáveis & Média \pm DP \\
\hline Idade (anos) & $48,80 \pm 7,08$ \\
\hline Altura (cm) & $161,16 \pm 8,18$ \\
\hline Peso (Kg) & $75,70 \pm 10,63$ \\
\hline \multirow[t]{2}{*}{ IMC (Kg/m²) } & $29,22 \pm 10,63$ \\
\hline & n (\%) \\
\hline \multicolumn{2}{|l|}{ Sexo } \\
\hline Feminino & $33(75,0)$ \\
\hline \multicolumn{2}{|l|}{ Estado Civil } \\
\hline Casado/união estável & $25(56,8)$ \\
\hline Solteiro & $13(29,5)$ \\
\hline Viúvo & $2(4,5)$ \\
\hline Separado/divorciado & $4(9,1)$ \\
\hline \multicolumn{2}{|l|}{ Anos de Estudo } \\
\hline Até 4 & $1(2,3)$ \\
\hline $5-8$ & $5(11,4)$ \\
\hline $9-11$ & $10(22,7)$ \\
\hline 12 ou mais & $28(63,6)$ \\
\hline \multicolumn{2}{|l|}{ Cor da Pele (IBGE) } \\
\hline Preta & $22(50,0)$ \\
\hline Parda & $19(43,2)$ \\
\hline Branca & $2(4,5)$ \\
\hline Amarela & $1(2,3)$ \\
\hline
\end{tabular}

Em relação ao fumo, 34 (77,3\%) relataram que nunca fumaram, 19 indivíduos $(43,2 \%)$ não consumiam bebida alcoólica, 36 sujeitos $(81,8 \%)$ estão em uso de medicação anti-hipertensiva regularmente, sendo a medicação mais utilizada por 26 (59,1\%) bloqueadores de receptores de angiotensina II, 25 indivíduos (56,8\%) estavam com a pressão arterial controlada, sendo que $28(63,6 \%)$ não praticam atividade física regular. Os indivíduos tiveram média PAS, PAD, FC, FR, SpO2 e percepção de esforço pela escala modificada de BORG no início do teste de $151,08 \pm 22,40,91,52 \pm 14,68,74,87 \pm 11,04,25,3 \pm 4,59,96,73 \pm 1,90$ e 3,11 $\pm 2,30$, respectivamente (Tabela 2 ). 


\begin{tabular}{|c|c|c|}
\hline Variáveis & & n (\%) \\
\hline \multicolumn{3}{|l|}{ Hábitos de Vida e Saúde } \\
\hline \multicolumn{3}{|l|}{ Tabagismo } \\
\hline Nunca fumou & & $34(77,3)$ \\
\hline Fumante & & $1(2,3)$ \\
\hline Ex-fumante & & $9(20,5)$ \\
\hline \multicolumn{3}{|l|}{ Consumo de Bebidas Alcoólicas } \\
\hline $\operatorname{Sim}$ & & $19(43,2)$ \\
\hline \multicolumn{3}{|l|}{ Medicamento Anti-hipertensivo } \\
\hline $\operatorname{sim}$ & & $36(81,8)$ \\
\hline \multicolumn{3}{|l|}{ Tipo de Medicação } \\
\hline Diurético tiazídico & & $15(34,1)$ \\
\hline Betabloqueador & & $15(34,1)$ \\
\hline Inibidores da ECA & & $2(4,5)$ \\
\hline BRA & & $26(59,1)$ \\
\hline Bloqueadores dos canais de cálcio & & $8(18,2)$ \\
\hline AAS & & $4(9,1)$ \\
\hline \multicolumn{3}{|l|}{ Classificação da PA } \\
\hline Hipertensão controlada & & $25(56,8)$ \\
\hline \multicolumn{3}{|l|}{ Atividade Física Regular } \\
\hline Não & & $28(63,6)$ \\
\hline Parâmetros Clínicos TC6 & $\mathbf{n}$ & Média \pm DP \\
\hline PAS & & $151,08 \pm 22,40$ \\
\hline PAD & & $91,52 \pm 14,68$ \\
\hline FC & & $74,87 \pm 11,04$ \\
\hline FR & & $25,3 \pm 4,59$ \\
\hline $\mathrm{SpO}_{2}$ & & $96,73 \pm 1,90$ \\
\hline BORG & & $3,11 \pm 2,30$ \\
\hline Distância Percorrida - Homens & 11 & $541,03 \pm 54,71$ \\
\hline Distância Percorrida - Mulheres & 33 & $500 \pm 45,70$ \\
\hline Distância Percorrida & & $510,25 \pm 60,73$ \\
\hline Distância Prevista Enright e Sherrill & & $559,27 \pm 59,80$ \\
\hline
\end{tabular}


A distância percorrida pelos homens em média foi $541,03 \pm 54,71$ metros, as mulheres percorreram $500 \pm 45,70$ metros.Na comparação da distância percorrida com distância prevista as médias foram de 510,25 $\pm 60,73$ e 559,27士59,80 metros, respectivamente, não apresentando significância estatística (Tabela 2).

Tabela 3. Associação entre distância percorrida, categoria da pressão arterial, uso de betabloqueador e categoria da distância obtida. Salvador-Ba. 2018. $(n=44)$

\begin{tabular}{|c|c|c|c|}
\hline Variáveis & $\mathbf{n}$ & $\begin{array}{c}\text { Distância percorrida } \\
\text { Média } \pm \text { DP }\end{array}$ & p-Valor \\
\hline \multicolumn{4}{|l|}{ Categoria de Hipertensão * } \\
\hline Controlada & 25 & $508,83 \pm 56,1$ & 0,84 \\
\hline Não controlada & 19 & $512,11 \pm 44,11$ & \\
\hline \multicolumn{4}{|l|}{ Uso de Betabloqueador* } \\
\hline Sim & & $506,78 \pm 46,03$ & 0,75 \\
\hline Não & 29 & $512,04 \pm 53,7$ & \\
\hline \multicolumn{4}{|l|}{ Categoria da Distância Obtida * } \\
\hline Alcançou distância prevista & 9 & $539 \pm 80$ & 0,05 \\
\hline Não alcançou distância prevista & 35 & $503 \pm 38,6$ & \\
\hline
\end{tabular}

Os indivíduos com hipertensão controlada apresentaram média da distância percorrida de 508,83 $\pm 56,1$ metros e de $512,11 \pm 44,11$ metros para hipertensão não controlada. Considerando um possível efeito do betabloqueador na CF observou-se que os indivíduos que faziam uso de betabloqueador obtiveram uma média de 506,78 $\pm 46,03$ metros, e indivíduos que não faziam uso tiveram uma média de 512,04ะ53,7 metros, não apresentando significância estatística. A média da distância obtida nos indivíduos que não alcançaram os valores previstos foi de $503 \pm 38,6$ metros, sendo que a maioria dos indivíduos percorreram em média $86,3 \pm 7,2 \%$ da distância prevista (Tabela 3).

Nessa amostra houve predomínio de $28(63,6 \%)$ indivíduos que não praticam atividade física de forma regular. Sabendo-se que nessa população pode ocorrer uma diminuição da distância obtida no TC6, consequentemente redução da capacidade funcional, ocorrendo tal condição devido à inadequação do desempenho dos sistemas musculoesquelético, respiratório e cardiovascular, análise de subgrupo foi realizada para identificar se o sedentarismo poderia ser modificador de resultado. 
Tabela 4. Comparação de variáveis antropométricas e clínicas entre indivíduos sedentários e não sedentários. Salvador-Ba. 2018. (n=28)

\begin{tabular}{|c|c|c|c|}
\hline Variáveis & $\begin{array}{c}\text { Sedentários } \\
n=18\end{array}$ & $\begin{array}{c}\text { Não Sedentários } \\
n=10\end{array}$ & p-Valor \\
\hline \multicolumn{4}{|l|}{ Sexo } \\
\hline Feminino & $13(72,2 \%)$ & $8(80,0 \%)$ & 0,51 \\
\hline \multicolumn{4}{|l|}{ Tabagismo } \\
\hline Nunca & $13(72,2 \%)$ & $7(70,0 \%)$ & 0,60 \\
\hline Fumante & $1(5,6 \%)$ & $0(0,0 \%)$ & \\
\hline Ex-Fumante & $4(22,2 \%)$ & $3(30,0 \%)$ & \\
\hline \multicolumn{4}{|l|}{ Consumo de Bebidas Alcoólicas } \\
\hline $\operatorname{Sim}$ & $9(50 \%)$ & $5(50 \%)$ & 0,65 \\
\hline \multicolumn{4}{|l|}{ Medicamento Anti-hipertensivo } \\
\hline $\operatorname{sim}$ & $15(83,3 \%)$ & $7(70,0 \%)$ & 0,36 \\
\hline \multicolumn{4}{|l|}{ Tipo de Medicação } \\
\hline Diurético tiazídico & $5(27,8 \%)$ & $4(40,0 \%)$ & 0,40 \\
\hline Betabloqueador & $6(33,3 \%)$ & $2(20,0 \%)$ & 0,39 \\
\hline Inibidores da ECA & $1(5,6 \%)$ & $1(10,0 \%)$ & 0,60 \\
\hline BRA & $11(61,1 \%)$ & $4(40,0 \%)$ & 0,25 \\
\hline Bloqueadores dos canais de cálcio & $1(5,6 \%)$ & $1(10,0 \%)$ & 0,60 \\
\hline AAS & $1(5,6 \%)$ & $1(10,0 \%)$ & 0,60 \\
\hline
\end{tabular}

Classificação da PA

Hipertensão controlada

$11(61,1 \%) \quad 8(80,0 \%)$

0,28

ECA:Enzima Conversora de Angiotensina;BRA:Bloqueadores de receptores de angiotensina

II;AAS:Ácido Acetil Salicílico; n: Número de Indivíduos; p: Significância; *Qui_Quadrado;

As variáveis de interesse que poderiam apresentar algum fator modificador pelo sedentarismo estão dispostas nas tabelas 4 e 5, em que se observa que não houve diferença estatística entre sedentários e praticantes de atividade regular. 
Tabela 5. Comparação de variáveis relacionadas à Distância Percorrida entre indivíduos sedentários e não sedentários. Salvador-Ba. 2018. (n=28)

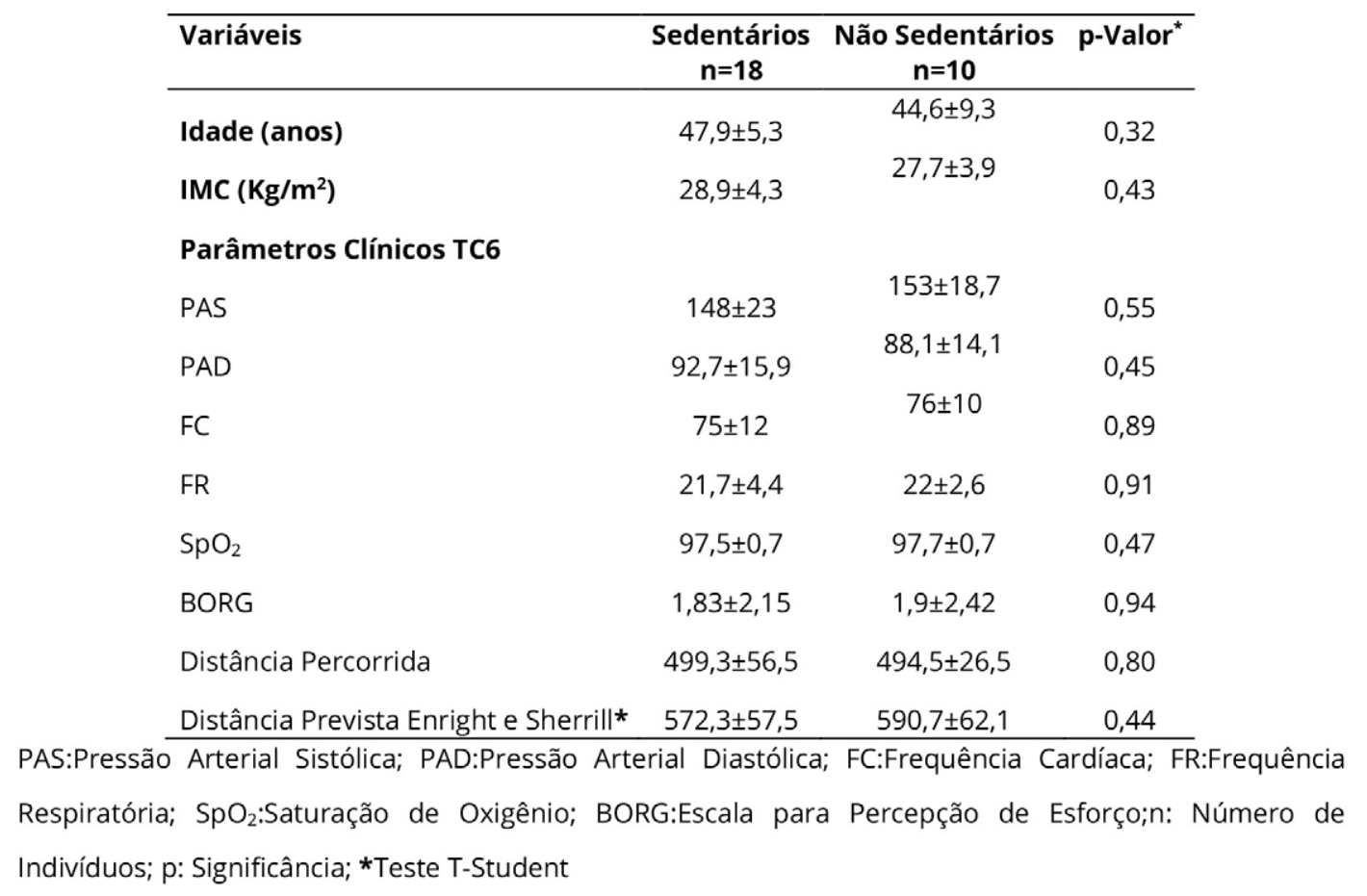

\section{Discussão}

Esta pesquisa teve como objetivo verificar a distância percorrida dos indivíduos hipertensos, apresentando resultados compatíveis com a literatura. Em geral, indivíduos com hipertensão já apresentam comprometimento da distância percorrida quando comparada com a distância prevista para cada pessoa, independente da pressão arterial estar controlada ou não. A maioria dos participantes (79,54\%) não conseguiram alcançar a distância prevista e o betabloqueador não foi fator interveniente para os resultados encontrados. No entanto, apesar dessa diferença os mesmos percorreram cerca de $86,3 \%$ da distância prevista,o que pode não apresentar relevância clínica.

Este fato pode estar associado ao aumento da resistência vascular periférica(RVP), e suas derivações. O aumento da RVP na hipertensão resulta de vasoconstricção arteriolar, o que pode ocasionar hipoperfusão muscular durante um tempo prolongado se o padrão for mantido. Em condições de restrição e aumento da demanda metabólica, a hipertensão pode ser um fator que ocasiona a redução do aporte nutricional muscular, podendo comprometer a contractilidade muscular, acometendo a capacidade funcional dos indivíduos ${ }^{8}$.

O estudo de Silveira et al. ${ }^{7}$, analisou 10 homens hipertensos com a média de idade de 48,9 $\pm 5,95$ anos, não prati-

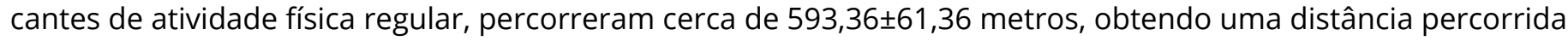
maior do que indivíduos do mesmo sexo, apesar da média de idade ser semelhante na presente investigação.

No estudo de Ribeiro et al. ${ }^{17}$, realizado com 15 mulheres hipertensas, com média de idade de $63,1 \pm 4$ anos, percorreram $468 \pm 4$ metros. Pedrosa et al. ${ }^{4}$, traz em seu estudo 32 mulheres hipertensas, com uma média de idade de $65,4 \pm 5,4$ anos, percorreram $428 \pm 85$ metros. Essas distâncias formam menores que a do presente estudo quando comparados com indivíduos do mesmo sexo. Ferreira et al. ${ }^{18}$, em seu estudo com 18 indivíduos hipertensos de ambos os sexos, faixa etária de 54 e 83 anos, percorreram 432,02 metros, obtendo uma distância percorrida menor que a da presente investigação, realizado com indivíduos de ambos os sexos, no entanto, a idade pode ser uma explicação para esse dado divergente. 
Sugere-se que modificações na capacidade funcional de indivíduos hipertensos tenham relação com os mecanismos que regulam as grandes e pequenas artérias $^{8}$. A resistência das arteríolas depende sobretudo do seu calibre, na hipertensão ocorre o estreitamento dos vasos, fazendo o coração aumentar a contractilidade muscular que regula a luz do vaso, acarretando em aumento da RVP, dificultando o transporte e a permeabilidade para a chegada energética e nutricional dos músculos ${ }^{8,19}$.

Em análise secundária, observou-se que a presença de maior número de sedentários nessa amostra não foi capaz de influenciar ou modificar os resultados referentes ao teste de caminhada. A análise dos resultados sugere que os valores da DP no TC6 abaixo dos valores previstos seja devido à condição cardiovascular.

A porcentagem dos valores da distância percorrida em relação à distância prevista podem não apresentar relevância clínica no presente estudo. Por outro lado, considerando a HAS fator de risco para doenças cardiovasculares, em que ocorre progressão sistêmica da morbidade, é importante refletir sobre um possível acompanhamento regular desses indivíduos, com avaliações sistematizadas da CF, para identificar o momento em que alterações da CF podem trazer repercussões clínicas significativas.

Baseado nesses dados sugere-se que indivíduos com hipertensão arterial sejam acompanhados regularmente e que o TC6 possa ser inserido como uma avaliação de prognóstico funcional, para que seja possível determinar diferença mínima clinicamente significante e um possível ponto de corte para prognóstico de comorbidades.

Esta investigação teve como limitação maior número de mulheres na amostra, uma vez que as mesmas têm a tendência a procurar o serviço de saúde em maior frequência em relação aos homens. $O$ estudo apresentou um baixo risco de viés, uma vez que não houve perda de participantes, da mesma maneira que não se obteve perda de dados dos desfechos principais. Os desfechos principais foram analisados de acordo com o proposto, bem como as variáveis com potencial modificação de desfecho.

\section{Conclusão}

Indivíduos com hipertensão arterial apresentam diminuição da distância percorrida, independente da pressão arterial estar controlada ou não.

\section{Contribuições das autoras}

Oliveira JS redigiu o manuscrito. Góes ALB contribuiu na orientação e auxílio da redação do manuscrito.

\section{Conflitos de interesses}

Nenhum conflito financeiro, legal ou político envolvendo terceiros (governo, empresas e fundações privadas, etc.) foi declarado para nenhum aspecto do trabalho submetido (incluindo mas não limitando-se a subvenções e financiamentos, participação em conselho consultivo, desenho de estudo, preparação de manuscrito, análise estatística, etc.).

\section{Referências}

1. Sociedade Brasileira de Cardiologia / Sociedade Brasileira de Hipertensão / Sociedade Brasileira de Nefrologia. VI Diretrizes Brasileiras de Hipertensão. Arq Bras Cardiol; 2010;95(1 supl.1):151. doi: $10.1590 / 50066-782 \times 2010001700001$

2. Goes ALB, Jesus DM, Silva TB, Lago VC, Souza LAP, Ladeia AMT. Influence of postural misalignment on blood pressure in hypertensive individuals : an exploratory cross sectional study. J Phys Res. 2018;8(2):248-257. doi: 10.17267/2238-2704rpf. v8i2.1955

3. DATASUS. Sistema de Informações Hospitalares do SUS (SIH/ SUS). [Internet]. 2018. Available from: http://www2.datasus.gov. br/DATASUS/index.php

\section{Pedrosa R, Holanda G. Correlação entre os testes da caminhada, marcha estacionária e TUG em hipertensas idosas. Rev Bras Fisioter. 2009;13(3):252-6. doi: 10.1590/S1413- $\underline{35552009005000030}$}

5. Accioly MF, Patrizzi LJ, Pinheiro PS, Bertoncello D, Walsh IAP. Exercícios físicos, mobilidade funcional, equilíbrio, capacidade funcional e quedas em idosos. ConScientiae Saúde. 2016;15(3):378-84. doi: 10.5585/ConsSaude.v15n3.6338

6. Ramos RA, Ferreira AS. Capacidade funcional de adultos com hipertensão avaliada pelo teste de caminhada de seis minutos: revisão sistemática. Fisioter Pesqui. 2014;21(3):257-63. doi: 10.590/1809-2950/66021032014 
7. Costa HS, Martins AMB, Quirino FC, Severino G, Novais LD, Lima MMO. Capacidade Funcional em Homens Hipertensos pela Distância Caminhada e Correlação com Valores Preditos. Fisioter Mov. 2009;22(4):557-64.

8. Soares VP, Dias AF, Jesus DM, Nascimento TS, Lago VC, Góes ALB. Correlação Entre Força Muscular E Capacidade Funcional Em Hipertensos. Rev Pesqui em Fisioter. 2016;6-15. doi: 10.17267/2238-2704rpf.v6i1.796

9. Meneghelo RS, Araújo CGS, Stein R, Mastrocolla LE, Albuquerque PF, Serra SM et al. III Guidelines of Sociedade Brasileira de Cardiologia on the exercise test. Arq Bras Cardiol. 2010;95(5 Suppl 1):1-26. doi: 10.1590/S0066-782X2010000800001

10. Soares, MR Pereira CAC. Teste de caminhada de seis minutos: valores de referência para adultos saudáveis no Brasil. J Bras Pneumol. 2011;37(5):576-83. doi: 10.1590/S2179$\underline{64912011000100004}$

11. Dias AF, Jesus DM, Negrão MC, Nascimento TS, Soares VP, Goes ALB,. Efeito da Palmilha de Reprogramação Postural na Capacidade Funcional em Hipertensos. Rev Pesqui em Fisioter. 2016;6(4):430-9. doi: 10.17267/2238-2704rpf.v6i4.1145

12. Malachias MVB, Souza W, Plavnik FL, Rodrigues C, Brandão A, Neves M et al. 7a Diretriz Brasileira De Hipertensão Arterial. Arq Bras Cardiol. 2016;107(3Supl.3):1-83.

13. ATS. Statement: Guidelines for the six-minute walk test. Am J Respir Crit Care Med. 2002;166(1):111-7. doi: 10.1164/ rccm.166/1/111

14. Schveitzer V, Claudino R, Ternes M. Teste de Caminhada de seis minutos: passos para realizá-lo. Rev Digit. 2009;14(137).

15. Enright PL, Sherrill DL. Reference equations for the six-minute walk in healthy adults. Am J Respir Crit Care Med. 1998;158(5 PART I):1384-7. doi: $10.1378 /$ chest.123.2.387

16. Dourado VZ. Reference Equations for the 6-Minute Walk Test in Healthy Individuals. Arq Bras Cardiol. 2011;96(6):128-38. doi: 10.1590/S0066-782X2011005000024

17. Ribeiro A, Younes C, Mayer D, Fréz AR, Riedi C. Teste de caminhada de seis minutos para avaliação de mulheres com fatores de risco cardiovascular. Fisioter em Mov. 2011;24(4):713-9. doi: 10.1590/S0103-51502011000400016

18. Ferreira KS, Carvalho SM, Alves MA. Correlação entre a distância alcançada no teste de caminhada de seis minutos e as variáveis idade e tempo de exercício em indivíduos hipertensos. Rev Func. 2009;2(2):100-8.

19. Krieger EM, Franchini KG, Krieger JE. Fisiopatogenia Da Hipertensão Arterial Pathophysiology of Primary Hypertension. Medicina (Ribeirao Preto Online). 1996;29(2/3):181-92. doi: 10.11606/issn.2176-7262.v29i2/3p181-192 